\title{
Características socio demográficas de las mujeres con abortos inducidos en un Hospital Público de Bucaramanga
}

\section{Socio-demographic characteristics of females who had undergone induced abortion in a public hospital in Bucaramanga}

Eliana M. Quintero-Roa ${ }^{1}$, Ricardo Ortiz-Serrano ${ }^{1}$, Miguel E. Ochoa-Vera $^{2}$, Mónika P. Consuegra-Rodríguez ${ }^{3}$ y Carol A. Oliveros ${ }^{3}$

\footnotetext{
1 Facultad de Ciencias de la Salud, Universidad Autónoma de Bucaramanga. Bucaramanga, Colombia.equintero@unab.edu.co,rortiz@unab.edu.co

2 Departamento de Salud pública, Escuela de Medicina. Universidad Industrial de Santander. Bucaramanga, Colombia. miguelenriqueochoavera@yahoo.es

3 Investigadores independientes
}

Recibido 28 Octubre 2009/Enviado para Modificación 15 Agosto 2010/Aceptado 8 Noviembre 2010

\section{RESUMEN}

Objetivo Analizar desde la perspectiva bioética de la justicia distributiva las características socio-demográficas de las mujeres que consultan por aborto provocado en el Hospital Local del Norte de Bucaramanga.

Métodos Se realizó un estudio descriptivo y transversal con una muestra por conveniencia, tomada entre las pacientes que consultaron en Urgencias de Ginecoobstetricia del Hospital Local del Norte de Bucaramanga entre el $1^{\circ}$ de marzo de 2008 y el 28 de febrero del 2009 con diagnóstico de aborto en cualquiera de sus presentaciones.

Resultados La muestra total fue de 93 pacientes de las cuales 16 aceptaron haberse inducido un aborto. Los factores asociados con este hecho fueron: pertenecer a un estrato socio-económico bajo, carecer de adecuada atención sanitaria y educativa en planificación familiar, carecer de pareja estable o que esta fuera disfuncional y que la pareja presionara inducir el aborto por motivos económicos o paternidad satisfecha. Un contraste de estos resultados con la legislación sanitaria vigente desde la perspectiva bioética de equidad en salud, revela que la aplicación de la normatividad es deficiente tanto en cobertura como en acciones educativas en salud sexual y reproductiva.

Conclusiones Facilitar a las pacientes que ejerzan su sexualidad de manera responsable y mejorar las oportunidades para acceder a mejores opciones económicas, puede disminuir la presencia de abortos provocados en la población que habita en la Comuna Norte de Bucaramanga.

Palabras Clave: Aborto inducido, equidad, planificación familiar, factores de riesgo. (fuente DeCS, BIREME) 


\section{ABSTRACT}

Objective To evaluate the socio-demographic characteristics of females who admitted to having previously undergone induced abortion who were attended at Hospital Local del Norte in Bucaramanga from the viewpoint of distributive justice.

Methods This was a descriptive, cross-sectional study of a convenience sample of patients who consulted the Hospital Local del Norte's Emergency GynaecologyObstetrics Service in Bucaramanga between March 1/2008 and February 28/2009 who had a diagnosis of abortion in any of their presentations.

Results The sample involved 93 patients, 16 of whom accepted that they had undergone an induced abortion. Factors associated with the event were belonging to a low socioeconomic stratum, lacking adequate health care and education in family planning, lack of a stable partner or that their relationship was dysfunctional and that the couple had pressured for an induced abortion for economic reasons or that their desire for parenting had already been satisfied. Contrasting these results with existing health legislation bioethics from a social justice in health viewpoint revealed that current legislation is deficient in both its coverage and extension to educational activity regarding sexual and reproductive health.

Conclusions Enabling patients to enjoy their sexuality responsibly and improving opportunities for access to better economic options may reduce induced abortions in the population living in Bucaramanga's northern commune

Key Words: Induced abortion, social justice, family planning, risk factor (source MeSH, $N M L)$.

$\mathrm{L}$ a clara relación que existe entre el ambiente social y la salud de una comunidad ha sido explicitada por múltiples publicaciones (1). Cuando esta relación está marcada por la presencia de la pobreza, se corre el riesgo de entrar en un círculo vicioso donde la pobreza genera mala salud y la mala salud mantiene la pobreza (2). En la actualidad, los efectos deletéreos que traen los déficits en la atención en salud sobre una comunidad, tienden a enfocarse como una cuestión de justicia social (2). Al analizar esta situación a la luz del concepto bioético de justicia, se concluye que es a la asistencia sanitaria desde el enfoque de la justicia distributiva a quien le compete interpretar esta problemática (3).

La teoría general de la justicia tiende a sustentarse en la definición de equidad, comprendida no como igualdad, sino como la valoración de las desigualdades (4) en la individualidad de una estructura social. Amartya Sen al exponer su idea de equidad en salud, propone hablar de "capacidades humanas" y de justicia distributiva capaz de brindar igualdad de oportunidades para desarrollar esas (5). 
Si así se plantea la equidad, Margareth Whitehead caracterizó la inequidad como las "diferencias que son innecesarias, evitables y además, consideradas injustas" (6). Entonces el problema no es que existan desigualdades, sino que las desigualdades pongan sistemáticamente en desventaja a un grupo (7). En salud, la presencia de inequidades se manifiesta como una diferencia en contra de la comunidad menos favorecida, que se expresa con resultados que van en detrimento de la salud y con una mayor presencia de enfermedad y muerte (8).

Una comunidad con déficits en la disponibilidad de recursos intelectuales y materiales para controlar su capacidad reproductiva, tiene pocas oportunidades para evitar una gestación no deseada o enfrentar el desafío de un embarazo (9). Países desarrollados, con legislaciones permisivas respecto del aborto provocado como Francia, Suiza, Italia y Holanda muestran índices de prevalencia de esta patología de entre 7,5 y 12 abortos inducidos por 1000 mujeres en edad reproductiva $(10,11)$, en contraposición con las cifras que muestran países pobres localizados en el África Subsahariana, América Latina y Asia sur oriental y central (12-17) que oscilan en promedio entre 30-40 abortos provocados por 1000 mujeres en edad reproductiva.

Es llamativo como cambian las estadísticas de los países desarrollados cuando la población se califica en consideración a características sociodemográficas que favorecen la inequidad (18). Por ejemplo, Dinamarca y Suecia reportan como factores de riesgo para generar abortos provocados, ser inmigrante proveniente de un país no occidental y perteneciente a un estatus socio-económico bajo (19), características que generan obvias dificultades lingüísticas, culturales y socio-económicas para acceder a mecanismos de planificación familiar (20-22).

En Colombia el empobrecimiento de un alto porcentaje de la población, la dependencia económica, la imposibilidad de generar riqueza y el desplazamiento interno forzado (23), han generado reestructuraciones culturales locales y nacionales, así como una intensificación de la exclusión y la intolerancia (24). Bucaramanga, capital de Santander, está habitada según el censo del año 2005 (25) por 516005 personas, de las cuales 125000 viven en la comuna $1 \mathrm{o}$ comuna norte. Dicha comuna se encuentra conformada por 25 barrios, 5 asentamientos y 2 urbanizaciones, todos clasificados en estratos socioeconómicos $0,1,2$ y 3 . En esta zona conviven comunidades receptoras socialmente deprimidas y desplazados por la violencia (26). De los 14600 hogares que forman la comuna norte, uno de cada tres tiene jefatura femenina y de estas jefes de hogar el $53 \%$ está desempleada (26). La información que 
poseemos sobre cuáles son las características socio-demográficas de esta comunidad y su nivel de influencia en el estadode salud es bajo (27). Ennuestro país, la principal investigación (28) que ha relacionado los factores sociales con la posibilidad de abortos inducidos, se centró en poblaciones predominantemente urbanas, de las principales capitales y fue desarrollada en la década de los 90 . Consideramos que las particulares características socio-demográficas ya descritas de la comuna norte de Bucaramanga ameritan un enfoque particular de esta problemática.

\section{METODOLOGÍA}

Este proyecto fue avalado por el Comité de Ética de la Investigación de la Facultad de Medicina de la Universidad Autónoma de Bucaramanga. Se trata de un estudio cuantitativo, descriptivo, transversal con una muestra por conveniencia tomada entre las pacientes que consultaron al Servicio de Urgencias de Gineco-obstetricia del Hospital Local del Norte (HLN) entre el $1^{\circ}$ de marzo de 2008 y el 28 de febrero del 2009, a quienes el médico tratante les realizó cualquiera de los diagnósticos identificados entre los códigos O021-O069 según la Clasificación Estadística internacional de Enfermedades (CIE 10) (29) (que corresponden a los diagnósticos de aborto completo oincompleto y sus asociaciones con cualquiera de sus complicaciones) y que aceptaron contestar la encuesta anónima. Se usó esta metodología para evitar el riesgo de implicaciones legales para los investigadores, dado que se trataba de evaluar pacientes que se indujeron un aborto ilegal.

Criterios de inclusión: Saber leer y escribir.

Se les explicó a las pacientes el objetivo del estudio y se les solicitó llenar de manera voluntaria una encuesta auto-administrada de forma anónima. Posteriormente, la paciente debía depositar el instrumento en una urna sellada localizada en un sitio accesible y privado dentro del área locativa del servicio de Urgencias de Gineco-obstetricia. Se consideró como población de estudio todas aquellas pacientes que posterior a recibir la explicación dada por parte del personal médico tratante al respecto, aceptaran participar de manera autónoma llenando el formulario. A los datos recolectados se les realizaron análisis univariados, bivariados, pruebas de Chi cuadrado y test exacto de Fischer según necesidad. Los análisis estadísticos se realizaron en el programa STATA 9.0.

\section{RESULTADOS}

Se recolectaron 93 encuestas de pacientes con diagnóstico de aborto, 16 de las cuales aceptaron haberlo inducido. El promedio de edad fue de 24,7 años, con 
extremos entre los 14 y 40 años. El número promedio de hijos por paciente fue de 1.9. No se encontraron diferencias significativas al respecto entre los grupos de aborto no provocado y aborto provocado. La población estaba compuesta principalmente por mujeres pertenecientes a estratos socio económicos bajos y dependientes económicamente (Tabla 1).

Tabla 1. Características socio demográficas categóricas de las pacientes con aborto provocado vs. no provocado

\begin{tabular}{|c|c|c|c|c|}
\hline & No provoc (\%) & Provoc (\%) & Total $(\%)$ & $P$ \\
\hline $\begin{array}{l}\text { Estrato Social } \\
0 \\
1 \\
2 \\
3 \\
\text { Total }\end{array}$ & $\begin{array}{c}3(4.0) \\
41(54.7) \\
26(34.6) \\
5(6.6) \\
75\end{array}$ & $\begin{array}{c}0(0.0) \\
6(37.5) \\
10(62.5) \\
0(0.0) \\
16\end{array}$ & $\begin{aligned} & 3(3.3) \\
& 47(51.7) \\
& 36(39.6) \\
& 5(5.5) \\
& 91\end{aligned}$ & 0,3 \\
\hline $\begin{array}{l}\text { Ocupación } \\
\text { Ninguna } \\
\text { Hogar } \\
\text { Estudia } \\
\text { Trabaja } \\
\text { Total }\end{array}$ & $\begin{aligned} & 3(3.9) \\
& 38(49.4) \\
& 7(9.1) \\
& 29(37.7) \\
& 77\end{aligned}$ & $\begin{array}{c}0(0.0) \\
6(37.5) \\
1(6.3) \\
9(56.2) \\
16\end{array}$ & $\begin{aligned} & 3(3.2) \\
& 44(47.3) \\
& 8(8.6) \\
& 38(40.9) \\
& 93\end{aligned}$ & 0,66 \\
\hline $\begin{array}{l}\text { Estado Civil } \\
\text { Soltera } \\
\text { Unión Libre } \\
\text { Casada } \\
\text { Viuda } \\
\text { Total }\end{array}$ & $\begin{array}{c}22(28.6) \\
48(62.3) \\
6(7.8) \\
1(1.3) \\
77\end{array}$ & $\begin{array}{c}6(37.5) \\
9(56.2) \\
0(0.0) \\
1(0.0) \\
16\end{array}$ & $\begin{array}{c}28(30.1) \\
57(61.2) \\
6(6.4) \\
2(2.1) \\
93\end{array}$ & 0,36 \\
\hline $\begin{array}{l}\text { Escolaridad } \\
\text { Primaria Incompleta } \\
\text { Primaria Completa } \\
\text { Secundaria Incompleta } \\
\text { Secundaria Completa } \\
\text { Universitaria Incompleta }\end{array}$ & $\begin{array}{c}7(9.5) \\
20(27.4) \\
24(32.8) \\
20(27.4) \\
2(2.7)\end{array}$ & $\begin{array}{l}3(18.7) \\
3(18.75) \\
8(50) \\
2(12.5) \\
0(0.0)\end{array}$ & $\begin{array}{c}10(11.2) \\
23(25.8) \\
32(35.9) \\
22(24.7) \\
2(2.2)\end{array}$ & 0,41 \\
\hline $\begin{array}{l}\text { Vive Con } \\
\text { Padres } \\
\text { Amigos } \\
\text { Pareja } \\
\text { Sola } \\
\text { Otros Familiares } \\
\text { Total }\end{array}$ & $\begin{aligned} & 16(20.7) \\
& 3(3.9) \\
& 50(64.9) \\
& 4(5.1) \\
& 4(5.1) \\
& 77\end{aligned}$ & $\begin{array}{c}6(37.5) \\
0(0.0) \\
8(50) \\
1(6.2) \\
1(6.2) \\
16\end{array}$ & $\begin{aligned} & 22(23.6) \\
& 3(3.2) \\
& 58(62.3) \\
& 5(5.3) \\
& 5(5.3) \\
& 93\end{aligned}$ & 0,48 \\
\hline $\begin{array}{l}\text { Depende de } \\
\text { otros familiares } \\
\text { Padres } \\
\text { Pareja } \\
\text { Usted misma }\end{array}$ & $\begin{array}{c}1(1.3) \\
4(5.1) \\
53(68.8) \\
19(24.7)\end{array}$ & $\begin{array}{c}0(0.0) \\
3(18.8) \\
7(43.7) \\
6(37.5)\end{array}$ & $\begin{array}{c}1(1.08) \\
7(7.5) \\
60(64.5) \\
25(26.9)\end{array}$ & 0.09 \\
\hline TOTAL & 77 & 16 & 93 & \\
\hline
\end{tabular}

El $85 \%$ de la población tuvo acceso al Régimen de Seguridad Social en Salud a pesar de lo cual, la proporción de pacientes que había recibido asesoría calificada por personal de salud entrenado en planificación fue solo del 27,3\%. Aunque un $57 \%$ de las pacientes consideró de manera subjetiva que su nivel de conocimientos en planificación era "bueno", esta apreciación es cuestionable si se contrasta con los diversos sitios donde fue asesorada (la casa, los amigos, 
la familia) y con la presencia de un no despreciable grupo de pacientes que no planificaba por "contraindicaciones médicas" o por "cansancio" (Tabla 2).

Tabla 2. Conocimiento, uso y causa de abandono de métodos de planificación familiar

\begin{tabular}{|c|c|c|c|c|}
\hline Asesoría en planificación & No Provoc (\%) & $\begin{array}{c}\text { Provoc } \\
(\%)\end{array}$ & $\begin{array}{c}\text { Total } \\
(\%)\end{array}$ & $p$ \\
\hline $\begin{array}{l}\text { No } \\
\text { Si } \\
\text { Total }\end{array}$ & $\begin{array}{c}7(9.1) \\
70(90.9) \\
77\end{array}$ & $\begin{array}{c}0(0.0) \\
16 \\
16\end{array}$ & $\begin{array}{c}7(7.5) \\
86 \\
93\end{array}$ & 0.2 \\
\hline $\begin{array}{l}\text { Nivel de conocimiento } \\
\text { Bueno } \\
\text { Regular } \\
\text { Malo } \\
\text { Total }\end{array}$ & $\begin{array}{c}31(59.6) \\
17(32.7) \\
4(7.7) \\
52\end{array}$ & $\begin{array}{l}8(50.0) \\
6(37.5) \\
2(12.5) \\
16\end{array}$ & $\begin{array}{c}39 \\
23 \\
6(8.8) \\
68\end{array}$ & 1.0 \\
\hline $\begin{array}{l}\text { Donde recibió información } \\
\text { Casa } \\
\text { Colegio } \\
\text { Amigos } \\
\text { Familiares } \\
\text { Institución de salud } \\
\text { Total }\end{array}$ & $\begin{aligned} & 14(18.9) \\
& 27(36.5) \\
& 11(14.9) \\
& 4(5.4) \\
& 18(24.3) \\
& 74\end{aligned}$ & $\begin{array}{c}4(28.6) \\
2(14.3) \\
0(0.0) \\
2(14.3) \\
6(42.9) \\
14\end{array}$ & $\begin{array}{c}18 \\
29(33) \\
11 \\
6(6.8) \\
24 \\
88\end{array}$ & 0.5 \\
\hline $\begin{array}{l}\text { Planificaba actualmente } \\
\text { No } \\
\text { Si } \\
\text { Total }\end{array}$ & $\begin{array}{c}69(89.6) \\
8(10.4)\end{array}$ & $\begin{array}{c}14(87,5) \\
2(12.5)\end{array}$ & $\begin{array}{l}83 \\
10 \\
93\end{array}$ & 0.68 \\
\hline $\begin{array}{l}\text { Razón para no planificar } \\
\text { Cansancio } \\
\text { Deseo embarazo } \\
\text { Falta de recursos } \\
\text { Pareja no permite planificación } \\
\text { Otro } \\
\text { Total }\end{array}$ & $\begin{array}{c}13(23.2) \\
23(41.1) \\
6(10.7) \\
4(7.1) \\
10(17.8) \\
56\end{array}$ & $\begin{array}{c}2(16.7) \\
0(0.0) \\
4(33.3) \\
2(16.7) \\
4(33.3) \\
12\end{array}$ & $\begin{array}{c}15 \\
23 \\
10 \\
6(8.8) \\
14(20) \\
68\end{array}$ & $\begin{array}{l}< \\
0.01\end{array}$ \\
\hline $\begin{array}{l}\text { Otros corresponde a: } \\
\text { Enfermedad o contraindicación médica } \\
\text { Ausencia de compañero estable } \\
\text { No le gusta planificar } \\
\text { Desconocimiento de métodos } \\
\text { Expectativa por otro método Planifica- }\end{array}$ & $\begin{array}{l}3(30.0) \\
1(10.0) \\
4(40.0) \\
1(10.0) \\
1(10.0)\end{array}$ & $\begin{array}{l}2(50.0) \\
2(50.0) \\
0(0.0) \\
0(0.0) \\
0(0.0)\end{array}$ & $\begin{array}{ll} & 5 \\
& 3 \\
& 4 \\
1 & (7.1) \\
1 & (7.1) \\
\end{array}$ & \\
\hline TOTAL & 10 & 4 & 14 & \\
\hline
\end{tabular}

Las razones de índole económica fueron las más frecuentemente usadas para explicar el rechazo hacia el embarazo (por parte de la paciente y de la pareja) y para coaccionar a la paciente a que se indujera el aborto (Tablas 3 y 4 .)

No tener una buena relación de pareja, asociado con el hecho de que la pareja no desee el embarazo y que ejerza presión para terminar la gestación fueron los ítems de mayor peso en las pacientes con aborto provocado (Tabla 4.) 
Tabla 3. Deseo del embarazo por parte de la paciente

\begin{tabular}{lcccc}
\hline \multicolumn{1}{c}{$\begin{array}{c}\text { Embarazo deseado o no } \\
\text { deseado }\end{array}$} & $\begin{array}{c}\text { No Provoc } \\
(\%)\end{array}$ & $\begin{array}{c}\text { Provocado } \\
(\%)\end{array}$ & $\begin{array}{c}\text { Total } \\
(\%)\end{array}$ & $P$ \\
Embarazo deseado & & & & \\
No & $10(13.51)$ & $15(93.75)$ & $25(27.78)$ & $<0.01$ \\
Si & $64(86.49)$ & $1(6.25)$ & $65(72.22)$ & \\
Total & 74 & 16 & $90(100)$ & \\
Motivo para no desear & & & & \\
Falta de apoyo familiar & $1(15.5)$ & $1(9.1)$ & $2(10.5)$ & \\
Falta apoyo de la pareja & $2(25.0)$ & $0(0.0)$ & $2(10.5)$ & 0.2 \\
Momento inadecuado de la vida & $0(0.0)$ & $3(27.3)$ & $3(15.8)$ & \\
Razones económicas & $5(62.5)$ & $6(54.6)$ & $11(57.9)$ & \\
Riesgo de malformaciones & $0(0.0)$ & $1(9.1)$ & $1(5.3)$ & \\
\hline
\end{tabular}

Tabla 4. Relación con la pareja, familiares y amigos

\begin{tabular}{|c|c|c|c|c|}
\hline Variable & No Provoc & Provocado & Total $(\%)$ & $P$ \\
\hline $\begin{array}{l}\text { Pareia estable } \\
\text { No } \\
\text { Si } \\
\text { Total }\end{array}$ & $\begin{array}{c}5(6.8) \\
70(93.3) \\
75\end{array}$ & $\begin{array}{c}5(31.2) \\
11(68.7) \\
16\end{array}$ & $\begin{array}{c}10(11) \\
81(89) \\
91(100)\end{array}$ & 0.01 \\
\hline \multicolumn{5}{|l|}{ Calidad relación } \\
\hline Buena & $58(84)$ & $3(25)$ & $61(75.3)$ & \\
\hline Regular & $10(14.5)$ & $6(50)$ & $16(19.7)$ & $<0.01$ \\
\hline Mala & $1(1.4)$ & $3(25)$ & $4(4.9)$ & \\
\hline Total & 69 & 12 & $81(100)$ & \\
\hline \multicolumn{5}{|l|}{ Pareja deseada } \\
\hline & $10(14.5)$ & $8(61.5)$ & $18(21.9)$ & $<0.01$ \\
\hline $\mathrm{Si}$ & $59(85.5)$ & $5(38.5)$ & $64(78)$ & \\
\hline Total & 69 & 13 & $82(100)$ & \\
\hline \multicolumn{5}{|l|}{ Motivo no deseo } \\
\hline Dudas de la paternidad & $1(20.0)$ & $0(0.0)$ & $1(7.7)$ & \\
\hline Finalización de la pareja & $1(20.0)$ & $0(0.0)$ & $1(7.7)$ & \\
\hline Miedo a asumir & $0(0.0)$ & $1(12.5)$ & $1(7.7)$ & 0.05 \\
\hline Paternidad satisfecha & $3(60.0)$ & $2(25.0)$ & $5(38.5)$ & \\
\hline Razones económicas & $0(0.0)$ & $5(62.5)$ & $5(38.5)$ & \\
\hline Total & 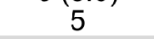 & 8 & $13(100.0)$ & \\
\hline \multicolumn{5}{|l|}{ Presión para abortar } \\
\hline No & $75(97.4)$ & $4(25.0)$ & $79(84.9)$ & $<0.01$ \\
\hline $\mathrm{Si}$ & $2(2.6)$ & $12(75.0)$ & $14(15.0)$ & \\
\hline \multicolumn{5}{|l|}{ Persona que presionó } \\
\hline Amigos & $1(1.3)$ & $7(43.8)$ & $8(57.1)$ & \\
\hline Pareja & $1(1.3)$ & $4(25.0)$ & $4(28.6)$ & $<0.01$ \\
\hline Familia & $0(0.0)$ & $1(6.3)$ & $2(14.3)$ & \\
\hline Total & 77 & 16 & $14(100)$ & \\
\hline \multicolumn{5}{|l|}{ Mecanismo de presión } \\
\hline Golpes & 0 & $2(28.6)$ & $2(28.6)$ & \\
\hline Total & 0 & 7 & $7(100.0)$ & \\
\hline
\end{tabular}

sanitaria en planificación familiar que evidencian estas pacientes, para describir dos de las principales características que estimulan inducirse un aborto. Si a lo anterior sumamos una pareja ausente o disfuncional que presiona la terminación de la gestación por razones económicas o paternidad satisfecha, queda configurado el perfil de características socio-demográficas que favorecen la posibilidad de abortos provocados en las pacientes usuarias del HLN. 


\section{DISCUSIÓN}

A pesar de que el tamaño de la muestra y la metodología cuantitativa utilizada son limitantes importantes para la evaluación a profundidad de la temática propuesta, los resultados obtenidos muestran con claridad hallazgos que ameritan ser debatidos y analizados. Es importante hacer énfasis en las cuestiones que competen a la planificación familiar. Es notorio apreciar la baja cobertura que muestra nuestra población en este indicador, máxime cuando la Norma Técnica para la Atención en Planificación Familiar a Hombres y Mujeres expedida por el Ministerio de la Protección Social en Marzo 3 de 2008 establece que "... Las siguientes actividades, procedimientos e intervenciones conforman las normas básicas mínimas que deben ser realizadas por las instituciones responsables de la atención en planificación familiar a hombres y mujeres, las cuales deben tener capacidad resolutiva y un equipo de salud capacitado para brindar atención integral humanizada y de calidad..." (30).

Genera serios cuestionamientos el hallazgo de diferencias estadísticamente significativas entre las pacientes de aborto no provocado Vs las de aborto provocado, que indican que una importante causa para no estar planificando es la falta de recursos económicos. El acuerdo 380 del 2007 (31) emitido por el Consejo Nacional de Seguridad Social en Salud, garantiza a toda la población de manera simétrica entre los Planes Obligatorios de Salud de los regímenes Subsidiado y Contributivo el acceso a métodos de planificación variados. Es clara la incongruencia que existe entre los hallazgos reportados y la teoría de la ley sanitaria. En asentamientos humanos como la comuna norte, se debería hacer un mejor y mayor énfasis en la oferta de acciones educativas efectivas en atención primaria en salud si queremos romper el círculo pobrezaenfermedad-pobreza. Aplicar mecanismos de control administrativo por parte del ente gubernamental que aseguren el desarrollo de la normativa vigente en función de una adecuada distribución de los recursos asignados para la atención de una población vulnerable, es un deber que no puede ser obviado por un sistema de salud democrático y justo. Volver la mirada hacia las Administradoras de Régimen Subsidiado para evaluar la forma y calidad con que se están desarrollando los procesos administrativos que soportan la atención primaria en planificación familiar, puede ser el primer paso para responder el gran interrogante que generan los extremadamente bajos índices de cobertura en este aspecto que se detectaron.

Otros aspectos de peso estadístico descritos como factores de riesgo para abortos inducidos fueron los motivos económicos como mecanismo de presión por parte de la pareja y de los amigos. Si retornamos al concepto de que no es justo que las inequidades pongan en desventaja a un grupo y enfocamos esa 
problemática para considerarla desde la perspectiva político-administrativa, podemos inferir que una mejoría en los ingresos y nivel de vida de esta población debería incidir en la disminución de la prevalencia de esta patología.

La responsabilidad de generar soluciones a estos problemas recae no solo en el administrador municipal, sino también en las instituciones educativas, en las Organizaciones No Gubernamentales y la sociedad bumanguesa en general. Estas soluciones deberían dirigirse tanto a la implementación de políticas y programas sólidos que faciliten a esta comunidad la posibilidad de acceder a labores dignamente remuneradas, como a mejorar el acceso a programas educativos oportunos y de calidad en salud sexual y reproductiva.

Solo cuando poseamos un sistema de salud sustentado en lineamientos políticos basados en la equidad y la justicia distributiva, acompañado de gestores administrativos comprometidos con la eficiencia y la calidad, trabajando en firme sociedad con efectores sanitarios guiados por la racionalidad técnico-científica y la ética podremos acercarnos de manera importante a alcanzar el fino balance que le permita al sistema ser financieramente viable y sanitariamente efectivo

Agradecimientos: A la Universidad Autónoma de Bucaramanga, a todo el personal de Sala de Partos del HLN, y especialmente a los Doctores Zulma Barbosa, Oscar Serrano y Andrea Massey.

\section{REFERENCIAS}

1. Marmot M. Improvement of social environment to improve health. Lancet; 1998;351:57-60.

2. Wastagff A. Pobreza y desigualdades en el sector de la salud. Rev Panam Salud Publica/Pan Am J Public Health 2002; 316-326.

3. Gracia D. ¿Qué es un sistema justo de servicios de salud? Principios para la asignación de recursos escasos. Biblioteca Virtual en Salud 1990; 527:187-200.

4. Hernandez-Alvarez M. El concepto de equidad y el debate sobre lo justo en salud. Rev. salud pública (Bogotá) 2008; 10 sup (1): 72-82.

5. Sen A. ¿Porqué la equidad en salud? Rev Panam Salud Publica/Pan Am J Public Health 2002; 11(5/6): 302-309.

6. Whitehead M. The concepts and principles of equity in health. Int J Health Serv 1992; 22: 429445.

7. Gómez E. Equidad, género y salud: retos para la acción. Rev Panam Salud Publica/Pan Am J Public Health 11(5/6), 2002: 454-461.

8. Martínez RM. Equidad en salud: Un análisis desde la mortalidad diferencial por régimen de aseguramiento. [Internet] Disponible en http://www.alapop.org/2009/images/PDF/ ALAP2004/_378.PDF. Consultado Mayo 2009.

9. Font-Ribera L, Pérez G, Salvador J, Borrell C. Socioeconomic Inequalities in unintended pregnancy and abortion decision. Journal of Urban Health: Bulletin of the New York Academy of Medicine 2007; 85(1): 125-135.

10. Benagiano G, Pera A. Decreasing the need for abortion: challenges and constraints. Int $\mathrm{J}$ Gynaecol Obstet 2000; 70(1):35-48. 
11. Henshaw S. Abortion laws and practice worldwide. In: Abortion Matters-Proceedings of the International Conference on Reducing the Need and Improving the Quality of Abortion Services. Netherlands: Uttrecht; 1997.

12. Department of Reproductive Health and Research, World Health Organization; Unsafe abortion: global and regional estimates of incidence of unsafe abortion and associated mortality in 2003. Geneva; 2007.

13. Menezes G, Estela A, Oliveira da Silva M. Induced abortion during youth: social inequalities in the outcome of the first pregnancy. Cad. Saúde Pública. 2006; 22(7):1431-1446.

14. Cabezas A, Langer A, Alvarez L, Bustamante P. Perfil socio demográfico del aborto inducido. Salud Pública de México 1998; 40(3):265-70.

15. Anselmo M, Caracalho D. Fatores de risco e preditores para o aborto induzido: estudo da base populacional. Cad. Saúde Públ 2006; 22(2):365-74.

16. Fonseca W, Misado Ch, Correia L, Parente J, Chagas F. Determinantes do aborto provocado entre mulheres admitidas em hospitais em localidade da região nordeste do Brasil. Rev. Saúde Públ 1996; 30

17. Briozzo L, León I, Rodríguez F, Vidiella G, Ferreiro G, Pons JE. Unsafe abortion in Uruguay. Int J Obstet Gynecol. 2004; 85 (1):70-3.

18. Briozzo L. Aborto provocado: un problema humano. Perspectivas para su análisis-Estrategias para su reducción. Rev Med Uruguay 2003; 19: 188-200.

19. Rash V, Gammeltoft T, Knudsen L, Tobiassen Ch, Ginzen A, Kempf L. Induced abortion in Denmark: effect of socio-economic situation and country of birth. European Journal of Public Health 2007; 18 (2): 144-149.

20.Vibeke R, Knudsen LB, Gammeltoft T, Christensen JT, Erenbjerg M, PlatzChristensen JJ, et al. Contraceptive attitudes and contraceptive failure among women requesting induced abortion in Denmark. Human Reproduction 2007; 22 (5): 1320-1326.

21. Holstrom L, Odlind V, Zatterstrom C, Johansson M, Granath F, Correia N, et al. Abortion rate and contraceptive practices in immigrant and native women in Sweden-Scandinavian. Journal of Public Health. 2003;31(6):405-10.

22. Bajos N, Lamarche-Vadel A, Gilbert F, Ferrand M, COCON Group, Moreau C. Contraception at the time of abortion: high-risk time or high-risk women? Human Reproduction 2006; 21 (11): 2862-2867.

23. Rodríguez CE. Aportes a la Evaluación de la Reforma de Salud en Colombia. Bioética, Justicia y Salud. Colección Bios y Ethos. Bogotá: Universidad El Bosque; 2006. pp 41-121.

24. Naranjo G. El desplazamiento forzado en Colombia. Reinvención de la identidad e implicaciones en las culturas locales y nacionales. Scripta Nova. Universidad de Barcelona 2001; 94 (1).

25. DANE [Internet]. Censo General de la República de Colombia 2005. [Internet] Disponible en: http://www.dane.gov.co/censo/ Consultado: Marzo de 2009.

26. Arias S, Gaviria MB. Salud y Desplazamiento en Colombia. Módulo 3 Bucaramanga. Organización Panamericana de la Salud-Universidad de Antioquia; 2003.

27. Ortiz R, Anaya NI, Sepúlveda C, Torres SJ, Camacho P. Caracterización de las adolescentes gestantes de Bucaramanga, Colombia: Un estudio transversal. MedUNAB 2005; 8 (2): 71-77.

28. Zamudio L, Rubiano N, Wartenberg L. El Aborto Inducido. Centro de Investigaciones sobre Dinámica Social (CIDS) Universidad Externado de Colombia; 1995.

29. Organización Mundial de la Salud. Clasificación Internacional de Enfermedades CIE 10. Madrid: Organización Mundial de la Salud; 1992.

30. Ministerio de la Protección Social. Resolución 0769 de Marzo 3 de 2008. Diario Oficial Marzo 6 de 2008; 46923: 15.

31. Consejo Nacional de Seguridad Social en Salud [Internet]. Acuerdo Número 380 de 2007 . Disponible en http://www.pos.gov.co/Documents/Archivos/normatividad_Regimen_Contributivo/ acuerdo 380 2007.pdf Consultado Mayo 2009. 